\title{
Adaptive Finite-Time Stabilization of High-Order Nonlinear Systems with Dynamic and Parametric Uncertainties
}

\author{
Meng-Meng Jiang and Xue-Jun Xie \\ Institute of Automation, Qufu Normal University, Shandong 273165, China \\ Correspondence should be addressed to Meng-Meng Jiang; jmm725@163.com
}

Received 1 May 2016; Accepted 31 July 2016

Academic Editor: Ricardo Aguilar-López

Copyright ( 2016 M.-M. Jiang and X.-J. Xie. This is an open access article distributed under the Creative Commons Attribution License, which permits unrestricted use, distribution, and reproduction in any medium, provided the original work is properly cited.

Under the weaker assumption on nonlinear functions, the adaptive finite-time stabilization of more general high-order nonlinear systems with dynamic and parametric uncertainties is solved in this paper. To solve this problem, finite-time input-to-state stability (FTISS) is used to characterize the unmeasured dynamic uncertainty. By skillfully combining Lyapunov function, sign function, backstepping, and finite-time input-to-state stability approaches, an adaptive state feedback controller is designed to guarantee high-order nonlinear systems are globally finite-time stable.

\section{Introduction}

Since the concept of finite-time stability was introduced in [1], many efforts have been made to study the problem of finitetime stabilization because of faster convergence rates, higher accuracies, and better disturbance rejection properties. Based on the finite-time stability theorem in [2-4], some finite-time stabilization results have been achieved by combining finitetime stability with backstepping design method, for example, [5-9] and the references therein.

Recently, more attention of finite-time stability has been focused on a family of high-order nonlinear systems of the form

$$
\begin{aligned}
& \dot{x}_{i}(t)=x_{i+1}^{p_{i}}(t)+\phi_{i}\left(x_{1}(t), \ldots, x_{i}(t), d\right), \\
& \quad i=1, \ldots, n-1, \\
& \dot{x}_{n}(t)=u^{p_{n}}(t)+\phi_{n}\left(x_{1}(t), \ldots, x_{n}(t), d\right),
\end{aligned}
$$

where $u \in R$ is the control input, $x=\left(x_{1}, \ldots, x_{n}\right)^{\top} \in R^{n}$ is the measured state, and $d \in R^{r}$ denotes the unknown parameter vector. For $i=1, \ldots, n, \phi_{i}: R^{i} \times R^{n_{0}} \times R^{+} \rightarrow R$ is an unknown and Lipschitz continuous function. $p_{i} \in R_{\text {odd }}^{\geq 1}:=\left\{p / q \in R^{+}: p\right.$ and $q$ are odd integers, $p \geq q\}$. System (1) is called high-order system if there exists at least $p_{i}>1, i \in\{1, \ldots, n\}$.
For system (1), when $d$ is known, $[10,11]$ studied finitetime stability, where the order of state $x_{j}(j=1, \ldots, i)$ in (10) and (11) can be taken value in $\left(0,1 /\left(p_{j} \ldots p_{i-1}\right)\right)$ with $p_{i} p_{i-1}=1$. The restrictive condition was relaxed by [12], in which all the states in the bounding condition were allowed to be of both low order and high order. When $d$ is unknown, it is well known that adaptive technique is an effective way to deal with control problem of nonlinear systems with parametric uncertainty. Reference [13] developed a continuous adaptive finite-time controller with the bounding condition of $\phi_{i}$ being an order equal to 1 . The latest paper [14] weakened the growth condition by allowing the order greater than 0 . However, there is no dynamic uncertainty considered by these papers.

The analysis and control problem of nonlinear systems with dynamic uncertainty have been an active research topic because dynamic uncertainty often arises from many different control engineering applications; see [15-19] and the references therein. In view of the benefits of finite-time convergence, finite-time stabilization of nonlinear systems with dynamic uncertainty has been regarded as one of the important issues. By characterizing dynamic uncertainty with finite-time input-to-state stability (FTISS), [20] constructed a finite-time adaptive state feedback controller for one-order nonlinear systems with dynamic and parametric uncertainties. Reference [21] gave the explicit definition of FTISS and 
developed a framework for the finite-time control analysis and synthesis based on FTISS. However, for more general high-order nonlinear systems, to the best of the authors' knowledge, no result on finite-time stabilization has been achieved until now.

Based on the above discussion, an interesting problem is put forward spontaneously: for more general high-order systems with dynamic and parametric uncertainties,

$$
\begin{aligned}
\dot{z}(t) & =\psi\left(z(t), x_{1}(t)\right), \\
\dot{x}_{i}(t) & =x_{i+1}^{p_{i}}(t)+\phi_{i}\left(x_{1}(t), \ldots, x_{i}(t), z(t), d\right), \\
& i=1, \ldots, n-1, \\
\dot{x}_{n}(t) & =u^{p_{n}}(t)+\phi_{n}\left(x_{1}(t), \ldots, x_{n}(t), z(t), d\right),
\end{aligned}
$$

$z \in R^{n_{0}}$ is the unmeasured state, referred to as dynamic uncertainty, $d \in R^{r}$ is the unknown parameter vector, $\phi_{i}$ : $R^{i} \times R^{n_{0}} \times R^{+} \rightarrow R(i=1, \ldots, n)$ is an unknown and Lipschitz continuous function, $\psi: R^{n_{0}} \times R \rightarrow R$ is piecewise continuous with respect to $z$ and Lipschitz continuous with respect to $x_{1}$, and $\psi(0,0)=0$; under the weaker assumption than Assumption 1 in [14] (see Remark 5 for a detailed discussion), can a finite-time stabilized continuous controller be designed?

In this paper, an affirmative solution to this problem is given. To solve this problem, finite-time input-to-state stability (FTISS) is used to characterize the unmeasured dynamic uncertainty. By skillfully combining Lyapunov function, sign function, backstepping, and FTISS approaches and overcoming some obstacles emerging in design and analysis owing to the relaxed condition on nonlinear functions, an adaptive state feedback controller is designed to guarantee high-order nonlinear system (2) is globally finite-time stable. An example demonstrates the theoretical result.

This paper is organized as follows. Section 2 gives preliminaries. Sections 3 and 4 provide the design and analysis of adaptive finite-time state feedback controller, following a simulation example in Section 5. Section 6 concludes the paper. The appendix proves Proposition 6 of Section 3.

\section{Mathematical Preliminaries}

Some notations and lemmas are to be used throughout this paper.

$R^{+}$stands for the set of all the nonnegative real numbers. For any vector $x=\left(x_{1}, \ldots, x_{n}\right)^{\top} \in R^{n}$, denote $\|x\|=$ $\left(\sum_{i=1}^{n} x_{i}^{2}\right)^{1 / 2}$. For $i=1, \ldots, n, \bar{x}_{i} \triangleq\left(x_{1}, \ldots, x_{i}\right)^{\top} \in R^{i}$. A function $f: R^{n} \rightarrow R$ is $\mathscr{C}$ if it is continuous and is $\mathscr{C}^{1}$ if it is continuously differential. $\mathscr{K}$ denotes the set of all functions: $R^{+} \rightarrow R^{+}$that are continuous, strictly increasing, and vanishing at zero, and $\mathscr{K}_{\infty}$ denotes the set of all functions that are of class $\mathscr{K}$ and unbounded. For simplicity, we sometimes denote a function $f(x(t))$ by $f(x)$ or $f$. Sign function $\operatorname{sgn}(x)$ is defined as $\operatorname{sgn}(x)=1$ if $x>0, \operatorname{sgn}(x)=0$ if $x=0$, and $\operatorname{sgn}(x)=-1$ if $x<0$.

Definition 1 (see [13]). Consider a system

$$
\dot{x}=f(x, t), \quad f(0, t)=0, x \in U_{0} \subset R^{n},
$$

where $f: U_{0} \times R^{+} \rightarrow R^{n}$ is continuous with respect to $x$ on an open neighborhood $U_{0}$ of the origin $x=0$. The equilibrium $x=0$ of the system is (local) finite-time stable if it is Lyapunov stable and finite-time convergent in a neighborhood $U \subseteq U_{0}$ of the origin. By "finite-time convergence" one means the following: if, for any initial condition $x\left(t_{0}\right)=x_{0} \in U$ at any given initial time $t_{0}$, there is a settling time $T>0$, such that every solution $x\left(t ; t_{0}, x_{0}\right)$ of system (3) is defined with $x\left(t ; t_{0}, x_{0}\right) \in U / 0$ for $t \in\left[t_{0}, T\right)$ and $\lim _{t \rightarrow T} x\left(t ; t_{0}, x_{0}\right)=0$, $x\left(t ; t_{0}, x_{0}\right)=0$ for any $t>T$. When $U=R^{n}$, the origin is a globally finite-time stable equilibrium.

$$
\begin{aligned}
& \text { Definition } 2 \text { (see [21]). Consider a system } \\
& \qquad \dot{x}=f(x, v), \quad f(0,0)=0, x \in R^{n}, v \in R^{m},
\end{aligned}
$$

where $v$ is the input and $f$ is continuous with respect to $(x, v)$. A continuous function $V(x)$ is called FTISS-Lyapunov function for system (4) if there exist $\mathscr{K}_{\infty}$-functions $\phi_{1}, \phi_{2}$, and $\phi_{3}$ and a positive constant $c$ such that

$$
\begin{aligned}
& \phi_{1}(\|x\|) \leq V(x) \leq \phi_{2}(\|x\|), \quad \forall x \in R^{n}, \\
& \dot{V}(x(t)) \leq-c V^{\alpha}(x(t))+\phi_{3}(\|v(t)\|), \quad 0<\alpha<1 .
\end{aligned}
$$

In the remainder of this section, we list several lemmas that serve as the basis for the design of state feedback controller for system (2). Lemma 3 is finite-time stability theorem. Lemmas $4-8$ are used to enlarge inequalities. Lemmas 9 and 10 are used to deal with sign function.

Lemma 3 (see [13]). Suppose that, for system (3), there is a $\mathscr{C}^{1}$ positive-definite function $V(x, t)$ (defined on $\widehat{U} \times R^{n}$, where $\widehat{U} \subset$ $U_{0} \subset R^{n}$ is a neighborhood of the origin), and a real number $c>0$ and $0<\alpha<1$, such that $\dot{V}(x, t)+c V^{\alpha}(x, t) \leq 0$ (along the trajectory) on $\widehat{U}$. Then $V(x, t)$ is locally finite-time convergent or equivalently becomes 0 locally in finite time, with its settling time $T \leq V^{1-\alpha}\left(x\left(t_{0}\right), t_{0}\right) / c(1-\alpha)$ for a given initial condition $x\left(t_{0}\right)$ in a neighborhood of the origin in $\widehat{U}$.

Lemma 4 (Young's inequality). Let real numbers $p \geq 1$ and $q \geq 1$ satisfy $1 / p+1 / q=1$; then for any $x, y \in R$ and any given positive number $\gamma>0, x y \leq \gamma|x|^{p}+(1 / q)(p \gamma)^{-q / p}|y|^{q}$.

Lemma 5 (Jensen's inequality). If $0<a_{1}<a_{2}$, then $\left(\sum_{i=1}^{n} x_{i}^{a_{2}}\right)^{1 / a_{2}} \leq\left(\sum_{i=1}^{n} x_{i}^{a_{1}}\right)^{1 / a_{1}}$ for any $x_{i} \geq 0, i=1, \ldots, n$.

Lemma 6 (see [22]). If $p \geq 1$, then $|x+y|^{1 / p} \leq|x|^{1 / p}+|y|^{1 / p}$, $|x+y|^{p} \leq 2^{p-1}\left|x^{p}+y^{p}\right|$ for any $x, y \in R$.

Lemma 7 (see [22]). If $p \in R_{\text {odd }}^{\geq 1}$, then $|x-y|^{p} \leq 2^{p-1}\left|x^{p}-y^{p}\right|$, $\left|x^{1 / p}-y^{1 / p}\right| \leq 2^{1-1 / p}|x-y|^{1 / p}$ for any $x, y \in R$.

Lemma 8 (see [23]). For a continuous function $f(x, y)$ with $x \in R^{m}, y \in R^{n}$, there exist smooth functions $a(x) \geq 0, b(y) \geq$ $0, c(x) \geq 1$, and $d(y) \geq 1$, such that $|f(x, y)| \leq a(x)+b(y)$, $|f(x, y)| \leq c(x) d(y)$.

Lemma 9 (see [24]). If $p=a / b \in R_{\text {odd }}^{\geq 1}, b \geq 1$, then $\left|x^{p}-y^{p}\right| \leq$ $\left.2^{1-1 / b}|\operatorname{sgn}(x)| x\right|^{a}-\left.\operatorname{sgn}(y)|y|^{a}\right|^{1 / b}$ for any $x, y \in R$. 
Lemma 10 (see [24]). $f(x)=\operatorname{sgn}(x)|x|^{a}$ is continuously differentiable, and $\dot{f}(x)=a|x|^{a-1}$, where $a \geq 1, x \in R$. Moreover, if $x=x(t), t \geq 0$, then $d f(x(t)) / d t=a|x(t)|^{a-1} \dot{x}(t)$.

\section{Finite-Time Convergence Analysis}

3.1. Problem Formulation and Assumptions. The purpose of this paper is to achieve a global finite-time control design for high-order nonlinear system (2) with dynamic and parametric uncertainties.

To achieve the purpose, we need the following assumptions.

Assumption 1. The $z$-subsystem has an FTISS-Lyapunov function $V_{0}(z)$ that satisfies

$$
\dot{V}_{0} \leq-c_{0} V_{0}^{\alpha_{0}}+\gamma_{0}\left(\left|x_{1}\right|\right),
$$

where $c_{0}>0,0<\alpha_{0}<1$ are constants, and $\gamma_{0}$ is a $\mathscr{K}_{\infty}$ function. Moreover, $\pi_{1}$ and $\pi_{2}$ are $\mathscr{K}_{\infty}$ functions such that

$$
\pi_{1}(\|z\|) \leq V_{0}(z) \leq \pi_{2}(\|z\|) .
$$

Assumption 2. For each $i=1, \ldots, n$, there is a constant $v \in$ $\left(0,1 / \sum_{j=1}^{n} p_{0} \cdots p_{j-1}\right)$ satisfying $(2-\nu) /\left(2-\nu-r_{n}\right)>2 \alpha_{0}$ and known $\mathscr{C}^{1}$ nonnegative function $\kappa_{1}$ and $\mathscr{C}$ nonnegative function $\kappa_{i 2}$ with $\kappa_{1}(0)=0$ and $\kappa_{i 2}(0, \ldots, 0)=0$ such that

$$
\begin{aligned}
& \left|\phi_{i}\left(x_{1}, \ldots, x_{i}, z, d\right)\right| \\
& \leq \kappa_{1}(\|z\|)+\gamma_{i}\left|x_{i+1}\right|^{p_{i}} \\
& \quad+\theta \kappa_{i 2}\left(x_{1}, \ldots, x_{i}\right) \sum_{j=1}^{i}\left|x_{j}\right|^{\left(r_{i}-v\right) / r_{j}+\mu_{i j}},
\end{aligned}
$$

where $\theta>0$ is an unknown constant, $\gamma_{i} \in[0,1), \mu_{i j} \in$ $[0,+\infty), p_{0}=1, x_{n+1}(t)=u(t)$, and

$$
\begin{aligned}
& r_{1}=1, \\
& r_{i}=\frac{r_{i-1}-v}{p_{i-1}}, \quad i=2, \ldots, n .
\end{aligned}
$$

Assumption 3. We assume that $\lim \sup _{s \rightarrow 0+}\left(\gamma_{0}(s) / s^{2}\right)<+\infty$ and $\lim \sup _{s \rightarrow 0+}\left(\kappa_{1}^{2}(s) / \pi_{1}(s)\right)<+\infty$.

Remark 4. Assumption 1 implies that $z$-subsystem is characterized by finite-time input-to-state stability (FTISS). The inequalities in Assumption 3 are the small-gain conditions.

Remark 5. The following discussions in order demonstrate that Assumption 2 encompasses and generalizes the existing results.

(i) When $z=0$ and $\theta$ is known, Assumption 2 includes the growth condition in [11]

$$
\begin{aligned}
\left|\phi_{i}\right| \leq \gamma_{i}\left(x_{1}, \ldots, x_{i}\right) \sum_{j=1}^{i}\left|x_{j}\right|^{\left(r_{i}+\tau\right) / r_{j}}, & \\
& \tau \in\left(-\frac{1}{\sum_{j=1}^{n} p_{0} \cdots p_{j-1}}, 0\right)
\end{aligned}
$$

as its special case (i.e., $\mu_{i j}=0$ in (8)), as well as the growth condition in [10]

$$
\left|\phi_{i}\right| \leq c \sum_{j=1}^{i}\left|x_{j}\right|^{\left(r_{i}+\tau\right) / r_{j}}, \quad \tau \in\left(-\frac{1}{\sum_{j=1}^{n} p_{0} \cdots p_{j-1}}, 0\right)
$$

as a special case (i.e., $\mu_{i j}=0$ and $\kappa_{i 2}=c$ is a constant).

From $v \in\left(0,1 / \sum_{j=1}^{n} p_{0} \cdots p_{j-1}\right), r_{1}=1, r_{i+1}=\left(r_{i}-v\right) / p_{i}$, it is easy to see that $0<\left(r_{i}-v\right) / r_{j}<1 /\left(p_{j} \cdots p_{i-1}\right)$, which implies that the power in condition (8) defined by $\left(r_{i}-\gamma\right) / r_{j}+$ $\mu_{i j}$ can take any value in an interval $(0,+\infty)$, while, for $[10,11]$, the powers only take values in $\left(0,1 /\left(p_{j} \cdots p_{i-1}\right)\right)$.

(ii) When $z=0$ and $\theta$ is unknown, (8) is reduced to Assumption 1 in [14]:

$$
\begin{aligned}
& \left|\phi_{i}\right| \leq \beta_{i}\left|x_{i+1}\right|^{p_{i}}+\theta \kappa_{i 2} \sum_{j=1}^{i}\left|x_{j}\right|^{\left(r_{i}+\omega\right) / r_{j}+\mu_{i j}}, \\
& \omega \in\left(-\frac{1}{\sum_{j=1}^{n} p_{0} \cdots p_{j-1}}, 0\right), \mu_{i j} \in[0,+\infty), \beta_{i} \in[0,1) .
\end{aligned}
$$

(iii) When $p_{i}=1$ for all $i=1, \ldots, n$, system (2) becomes

$$
\begin{aligned}
\dot{z} & =\psi\left(z, x_{1}\right), \\
\dot{x}_{i} & =x_{i+1}+\phi_{i}\left(x_{1}, \ldots, x_{i}, z, d\right), \quad i=1, \ldots, n-1, \\
\dot{x}_{n} & =u+\phi_{n}\left(x_{1}, \ldots, x_{n}, z, d\right),
\end{aligned}
$$

which is studied by $[20,21]$.

By the discussions, it is highlighted that this paper substantially extends the results of these papers; namely, for more general high-order nonlinear systems (2) with dynamic and parametric uncertainties, the finite-time control problem is to be solved under weaker condition (8).

3.2. Design of Adaptive Finite-Time Controller. In what follows, we denote $\sigma=\max \left\{\theta, \theta^{(2-\gamma) / r_{n}}\right\}$, which is unknown because $\theta$ is unknown, $\widehat{\sigma}(t)$ is the estimate of $\sigma$, and $\widetilde{\sigma}(t)=$ $\sigma-\widehat{\sigma}(t)$ is the estimation error. Denote $x=\left(x_{1}, \ldots, x_{n}\right)^{\top}$, $\bar{x}_{i}=\left(x_{1}, \ldots, x_{i}\right)^{\top} \in R^{i}, i=1, \ldots, n$. For simplicity, denote $[x]^{p}=|x|^{p} \operatorname{sgn}(x)$, where $x \in R, \operatorname{sgn}(\cdot)$ is sign function whose definition is in the notations explained in Section 2, $[x]^{p} \cdot x=|x|^{p+1}$, and $[x]^{p}$ is obviously $\mathscr{C}^{1}$ if $p \geq 1$.

To give the design of controller, we first define the parameters $\beta_{0}, \beta_{1}, \ldots, \beta_{n}$ recursively as

$$
\begin{aligned}
\beta_{0} & =1, \\
\left(\beta_{i} p_{i}+1\right) r_{i+1} & =\left(\beta_{i-1} p_{i-1}+1\right) r_{i}>0, \quad i=1, \ldots, n .
\end{aligned}
$$

From (14), it follows that

$$
\begin{aligned}
& 1<r_{i} \beta_{i-1} p_{i-1}<r_{i+1} \beta_{i} p_{i}, \\
& 1<\beta_{1} p_{1}<\cdots<\beta_{n} p_{n},
\end{aligned}
$$


Besides, from (15), it leads to $r_{i} p_{i-1}+r_{i-1} \beta_{i-2} p_{i-2}=r_{i+1} p_{i}+$ $r_{i} \beta_{i-1} p_{i-1}$; then

$$
\begin{aligned}
r_{i} p_{i-1}+r_{i-1} \beta_{i-2} p_{i-2}=r_{2} p_{1}+r_{1} \beta_{0} p_{0}=2- & \\
& i=1, \ldots, n .
\end{aligned}
$$

Secondly, we introduce the following coordinate transformation:

$$
\begin{aligned}
& \begin{array}{l}
w_{k}(t) \\
=\left[x_{k}(t)\right]^{\beta_{k-1} p_{k-1}}-\left[v_{k-1}\left(\bar{x}_{k-1}(t), \widehat{\sigma}(t)\right)\right]^{\beta_{k-1} p_{k-1}}, \\
k=1, \ldots, n,
\end{array} \\
& \begin{aligned}
u(t)=v_{n}(x(t), \widehat{\sigma}(t)), \\
v_{k}^{p_{k}}\left(\bar{x}_{k}(t), \widehat{\sigma}(t)\right) \\
=-\left[w_{k}(t)\right]^{\left(r_{k}-v\right) / r_{k} \beta_{k-1} p_{k-1}} \Phi_{k}\left(\bar{x}_{k}(t), \widehat{\sigma}(t)\right), \\
k=1, \ldots, n,
\end{aligned}
\end{aligned}
$$

where $\Phi_{k}, k=1, \ldots, n$, are $\mathscr{C}^{1}$ positive functions to be specified later. For the sake of consistency, we let $\Phi_{0}=0$ and $v_{0}=0$.

Finally, to obtain the detailed expression of $u$, we determine $\Phi_{1}, \ldots, \Phi_{n}$ by induction.

Initial Step. Consider

$$
\dot{x}_{1}=x_{2}^{p_{1}}+\phi_{1}\left(x_{1}, z, d\right) \text {. }
$$

Take $V_{1}\left(x_{1}\right)=x_{1}^{2} / 2$; then $V_{1}$ is $\mathscr{C}^{1}$ positive-definite and $\dot{V}_{1}\left(x_{1}\right)=x_{1}\left(x_{2}^{p_{1}}-v_{1}^{p_{1}}\right)+x_{1} v_{1}^{p_{1}}+x_{1} \phi_{1}$. By (15), (17), Lemmas 4 and 6, Assumption 2, and $\sigma \geq \theta$,

$$
\begin{aligned}
x_{1} \phi_{1} \leq & \left|x_{1}\right| \kappa_{1}(\|z\|)+x_{1} \cdot \gamma_{1}\left|x_{2}\right|^{p_{1}} \\
& +\left|x_{1}\right| \kappa_{12}\left(x_{1}\right)\left|x_{1}\right|^{1-\nu+\mu_{11}} \theta \\
\leq & \left|w_{1}\right|^{2-\nu}+\kappa_{1}^{(2-\nu) /(1-\nu)}+\theta \bar{\kappa}_{12}\left(x_{1}\right)\left|x_{1}\right|^{2-\nu} \\
& +\gamma_{1}\left|w_{1}\right|\left|w_{2}\right|^{1 / \beta_{1}}+\gamma_{1}\left|w_{1}\right|^{2-\gamma} \Phi_{1} \\
\leq & \left|w_{1}\right|^{2-\nu}\left(1+\sigma b_{1}\left(x_{1}\right)\right)+\kappa_{1}^{(2-\nu) /(1-v)} \\
& +\gamma_{1}\left|w_{1}\right|\left|w_{2}\right|^{1 / \beta_{1}}+\gamma_{1}\left|w_{1}\right|^{2-\nu} \Phi_{1},
\end{aligned}
$$

where $b_{1}\left(x_{1}\right)=\bar{\kappa}_{12}\left(x_{1}\right)=\left|x_{1}\right|^{\mu_{11}} \kappa_{12}\left(x_{1}\right)$ is a $\mathscr{C}$ nonnegative function and $b_{1}(0)=0$. By Lemma 8 , we choose a $\mathscr{C}^{1}$ positive function $\Phi_{1}\left(x_{1}, \widehat{\sigma}\right)$ dominating the following function:

$$
\begin{aligned}
\Phi_{1}^{0} & =\frac{1}{1-\gamma_{1}}\left(1+L_{1}+\frac{\gamma_{0}\left(\left|x_{1}\right|\right)}{\left|x_{1}\right|^{2-\nu}}\left(\mu\left(x_{1}\right)+1\right)\right. \\
& \left.+\widehat{\sigma} b_{1}\left(x_{1}\right)\right), \quad L_{1}>0,
\end{aligned}
$$

where $\mu\left(x_{1}\right)$ is a $\mathscr{C}^{1}$ function to be determined. Because of $v>0$ and Assumption 3, $\Phi_{1}^{0}$ is a continuous function. After some manipulations, we have

$$
\begin{aligned}
\dot{V}_{1}\left(x_{1}\right) \leq & -\gamma_{0}\left(\left|x_{1}\right|\right)\left(\mu\left(x_{1}\right)+1\right)-L_{1}\left|w_{1}\right|^{2-\gamma} \\
& +\kappa_{1}^{(2-\nu) /(1-\nu)}+\left|w_{1}\right|\left|x_{2}^{p_{1}}-v_{1}^{p_{1}}\right| \\
& +\left(\widetilde{\sigma}+\eta_{1}\right) \varphi_{1}-\eta_{1} \dot{\widehat{\sigma}}+\gamma_{1}\left|w_{1}\right|\left|w_{2}\right|^{1 / \beta_{1}}
\end{aligned}
$$

where $\eta_{1}=0, \varphi_{1}\left(x_{1}\right)=\left|x_{1}\right|^{2-v} b_{1}\left(x_{1}\right) \geq 0$, and $\dot{\hat{\sigma}}$ will be determined later.

Inductive Step. We give this step by the following proposition.

Proposition 6. Suppose at Step $i-1$, for system

$$
x_{j}=x_{j+1}^{p_{j}}+\phi_{j}\left(x_{1}, \ldots, x_{j}, z, d\right), \quad j=1, \ldots, i-1,
$$

there is a $\mathscr{C}^{1}$ function $V_{i-1}\left(\bar{x}_{i-1}, \widehat{\sigma}\right)$ which is positive-definite with respect to $x_{1}, \ldots, x_{i-1}$ and $\mathscr{C}^{1}$ positive function $\Phi_{i-1}$ such that

$$
\begin{aligned}
\dot{V}_{i-1}\left(\bar{x}_{i-1}, \widehat{\sigma}\right) \leq & -\gamma_{0}\left(\left|x_{1}\right|\right)\left(\mu\left(x_{1}\right)+1\right)-L_{i-1} Q_{i-1}^{2-\nu} \\
& +\left|w_{i-1}\right|\left|x_{i}^{p_{i-1}}-v_{i-1}^{p_{i-1}}\right| \\
& +\left(\widetilde{\sigma}+\eta_{i-1}\right) \varphi_{i-1}-\eta_{i-1} \dot{\widehat{\sigma}} \\
& +\sum_{j=1}^{i-1} \kappa_{1}^{(2-\nu) /\left(2-\gamma-r_{j} \beta_{j-1} p_{j-1}\right)} \\
& +\sum_{j=2}^{i-1} \kappa_{1}^{(2-\nu) /\left(2-\gamma-r_{j}\right)} \\
& +\gamma_{i-1}\left|w_{i-1}\right|\left|w_{i}\right|^{1 / \beta_{i-1}}, \quad L_{i-1}>0
\end{aligned}
$$

where

$$
\begin{aligned}
& Q_{i-1}\left(\bar{x}_{i-1}, \widehat{\sigma}\right)=\left(\left|w_{1}\right|^{2-\nu}+\left|w_{2}\right|^{(2-\nu) / r_{2} \beta_{1} p_{1}}+\cdots\right. \\
& \left.+\left|w_{i-1}\right|^{(2-\nu) / r_{i-1} \beta_{i-2} p_{i-2}}\right)^{1 /(2-\nu)}, \\
& \eta_{i-1}\left(\bar{x}_{i-1}, \widehat{\sigma}\right)=-\sum_{l=1}^{i-1} \frac{\partial W_{l}}{\partial \widehat{\sigma}}
\end{aligned}
$$

$\varphi_{i-1}\left(\bar{x}_{i-1}, \widehat{\sigma}\right) \geq 0$ is $\mathscr{C}^{1}$. Then, for system

$$
x_{j}=x_{j+1}^{p_{j}}+\phi_{j}\left(x_{1}, \ldots, x_{j}, z, d\right), \quad j=1, \ldots, i,
$$


the ith function $V_{i}\left(\bar{x}_{i}, \widehat{\sigma}\right)=V_{i-1}\left(\bar{x}_{i-1}, \widehat{\sigma}\right)+W_{i}\left(\bar{x}_{i}, \widehat{\sigma}\right)$ is $\mathscr{C}^{1}$ and positive-definite with respect to $x_{1}, \ldots, x_{i}$, and one can find a $\mathscr{C}^{1}$ positive function $\Phi_{i}$ (see (A.14) in the Appendix) such that

$$
\begin{aligned}
\dot{V}_{i}\left(\bar{x}_{i}, \widehat{\sigma}\right) \leq & -\gamma_{0}\left(\left|x_{1}\right|\right)\left(\mu\left(x_{1}\right)+1\right)-L_{i} Q_{i}^{2-\nu} \\
& +\left|w_{i}\right|\left|x_{i+1}^{p_{i}}-v_{i}^{p_{i}}\right|+\left(\widetilde{\sigma}+\eta_{i}\right) \varphi_{i}-\eta_{i} \dot{\widehat{\sigma}} \\
& +\sum_{j=1}^{i} \kappa_{1}^{(2-\nu) /\left(2-\nu-r_{j} \beta_{j-1} p_{j-1}\right)} \\
& +\sum_{j=2}^{i} \kappa_{1}^{(2-\nu) /\left(2-\nu-r_{j}\right)}+\gamma_{i}\left|w_{i}\right|\left|w_{i+1}\right|^{1 / \beta_{i}},
\end{aligned}
$$

where $L_{i}=((n-1) / n) L_{i-1}>0, \varphi_{i}\left(\bar{x}_{i}, \widehat{\sigma}\right)=\varphi_{i-1}\left(\bar{x}_{i-1}, \widehat{\sigma}\right)+$ $\left|w_{i}\right|^{(2-v) / r_{i} \beta_{i-1} p_{i-1}} b_{i}\left(\bar{x}_{i}, \widehat{\sigma}\right)$ (see (A.12) in the Appendix) is continuous, and

$$
W_{i}\left(\bar{x}_{i}, \widehat{\sigma}\right)=\int_{v_{i-1}}^{x_{i}}\left([s]^{\beta_{i-1} p_{i-1}}-\left[v_{i-1}\right]^{\beta_{i-1} p_{i-1}}\right) \mathrm{d} s,
$$

$$
1 \leq i \leq n \text {. }
$$

Proof. See the Appendix.

At Step $n$, (27) holds with $w_{n+1}=0$. Hence, by choosing $V_{n}(x, \widehat{\sigma})=V_{n-1}\left(\bar{x}_{n-1}, \widehat{\sigma}\right)+W_{n}(x, \widehat{\sigma})$, we can get

$$
u^{p_{n}}=v_{n}^{p_{n}}=-\left[w_{n}\right]^{\left(r_{n}-v\right) / r_{n} \beta_{n-1} p_{n-1}} \Phi_{n}(x, \widehat{\sigma})
$$

with an appropriate function $\Phi_{n}$ and

$$
\dot{\widehat{\sigma}}=-\dot{\widetilde{\sigma}}=\varphi_{n}
$$

such that

$$
\begin{aligned}
\dot{V}_{n}(x, \widehat{\sigma}) \leq & -\gamma_{0}\left(\left|x_{1}\right|\right)\left(\mu\left(x_{1}\right)+1\right)-L_{n} Q_{n}^{2-\nu}+\widetilde{\sigma} \varphi_{n} \\
& +\sum_{j=1}^{n} \kappa_{1}^{(2-\nu) /\left(2-\nu-r_{j} \beta_{j-1} p_{j-1}\right)} \\
& +\sum_{j=2}^{n} \kappa_{1}^{(2-\nu) /\left(2-\nu-r_{j}\right)}, \quad L_{n}>0 .
\end{aligned}
$$

\section{Finite-Time Convergence Analysis}

We state the main result in this paper.

Theorem 7. The solutions of the closed-loop system (2), (29), and (30) are bounded, and the trajectory $(z(t), x(t))$ is finitetime convergent to the origin $0 \in R^{n+n_{0}}$.

Proof. Define

$$
\begin{aligned}
\bar{\kappa}_{1}(s)= & \sum_{i=1}^{n} \kappa_{1}(s)^{(2-\nu) /\left(2-\nu-r_{i} \beta_{i-1} p_{i-1}\right)} \\
& +\sum_{i=2}^{n} \kappa_{1}(s)^{(2-\nu) /\left(2-\nu-r_{i}\right)}
\end{aligned}
$$

From Assumption $3, \kappa_{1}(s)$ is $\mathscr{C}^{1},(2-\nu) /\left(2-\nu-\beta_{0}\right)=1+1 /(1-$ $v)>2$, and, for $i=2, \ldots, n,(2-v) /\left(2-v-r_{i} \beta_{i-1} p_{i-1}\right)>2$, $(2-v) /\left(2-v-r_{i}\right)>2 \alpha_{0}$, it follows that

$$
\limsup _{s \rightarrow 0+} \frac{\bar{\kappa}_{1}(s)}{\pi_{1}^{\alpha_{0}}(s)}<+\infty
$$

The proof is divided into two parts.

Step 1. We show that the trajectories $(z(t), x(t), \widehat{\sigma}(t))$ of the closed-loop system (2), (29), and (30) are bounded.

Take a Lyapunov function as

$$
\begin{aligned}
V_{c}(x, z, \widehat{\sigma})=V_{n}(x, \widehat{\sigma})+V_{*}(z)+\frac{1}{2} \widetilde{\sigma}^{2}, & \\
V_{*}(z) & =\int_{0}^{V_{0}(z)} \rho(s) \mathrm{d} s,
\end{aligned}
$$

where $\rho: R^{+} \rightarrow R^{+}$is a continuous nondecreasing function with $\rho(0)>0$. It follows from (7) that when $\|z\|>$ $\left(\gamma_{0}\left(\left\|x_{1}\right\|\right) / c_{0} \epsilon\right)^{1 / \alpha_{0}},-c_{0} \epsilon \rho\left(V_{0}(z)\right) V_{0}^{\alpha_{0}}(z)+\rho\left(V_{0}(z)\right) \gamma_{0}\left(\left|x_{1}\right|\right)<$ 0 ; when $\|z\| \leq\left(\gamma_{0}\left(\left\|x_{1}\right\|\right) / c_{0} \epsilon\right)^{1 / \alpha_{0}}, \rho\left(V_{0}(z)\right) \gamma_{0}\left(\left|x_{1}\right|\right) \leq$ $\rho\left(\pi_{2}\left(\pi_{1}^{-1}\left(\left(\gamma_{0}\left(\left|x_{1}\right|\right) / c_{0} \epsilon\right)^{1 / \alpha_{0}}\right)\right)\right) \gamma_{0}\left(\left|x_{1}\right|\right)$. Then we observe from (30), (31), and (34) that

$$
\begin{aligned}
& \dot{V}_{c} \\
& \leq-\gamma_{0}\left(\left|x_{1}\right|\right)\left(\mu\left(x_{1}\right)+1\right)-L_{n} Q_{n}^{2-\gamma}+\bar{\kappa}_{1}(\|z\|) \\
& \quad-c_{0} \rho\left(V_{0}(z)\right) V_{0}^{\alpha_{0}}(z)+\rho\left(V_{0}(z)\right) \gamma_{0}\left(\left|x_{1}\right|\right) \\
& \leq-\gamma_{0}\left(\left|x_{1}\right|\right)\left(\mu\left(x_{1}\right)+1\right)-L_{n} Q_{n}^{2-\gamma}+\bar{\kappa}_{1}(\|z\|) \\
& \quad-c_{0}(1-\epsilon) \rho\left(V_{0}(z)\right) V_{0}^{\alpha_{0}} \\
& \quad+\rho\left(\pi_{2}\left(\pi_{1}^{-1}\left(\left(\frac{\gamma_{0}\left(\left|x_{1}\right|\right)}{c_{0} \epsilon}\right)^{1 / \alpha_{0}}\right)\right)\right) \gamma_{0}\left(\left|x_{1}\right|\right), \\
& \quad 0<\epsilon<1 .
\end{aligned}
$$

According to (33) and Lemma 8, we can find a desired function $\rho$ and a $\mathscr{C}^{1}$ function $\mu$ such that

$$
\begin{aligned}
& c_{0}(1-\epsilon) \rho\left(\pi_{1}(\|z\|)\right) \pi_{1}^{\alpha_{0}}(\|z\|) \geq 2 \bar{\kappa}_{1}(\|z\|), \\
& \mu\left(x_{1}\right)+1 \geq \rho\left(\pi_{2}\left(\pi_{1}^{-1}\left(\left(\frac{\gamma_{0}\left(\left|x_{1}\right|\right)}{c_{0} \epsilon}\right)^{1 / \alpha_{0}}\right)\right)\right) .
\end{aligned}
$$

Substituting (36) into (35) leads to

$$
\dot{V}_{c} \leq-L_{n} Q_{n}^{2-\nu}-\frac{\mathcal{c}_{0}(1-\epsilon)}{2} \rho\left(V_{0}(z)\right) V_{0}^{\alpha_{0}}(z) \leq 0,
$$

which implies the boundedness of $z(t), x(t)$, and $\widehat{\sigma}(t)$.

Step 2. We show that the trajectories $(z(t), x(t))$ are finitetime convergent.

Consider $\bar{V}_{c}(x, z, \widehat{\sigma})=V_{n}(x, \widehat{\sigma})+V_{*}(z)$, which is positivedefinite with respect to $(z, x)$. Then

$$
\dot{\bar{V}}_{c} \leq-L_{n} Q_{n}^{2-\nu}-\frac{c_{0}(1-\epsilon)}{2} \rho\left(V_{0}(z)\right) V_{0}^{\alpha_{0}}(z)+\widetilde{\sigma} \varphi_{n} .
$$


At first, we consider local finite-time convergence in a small neighborhood around $(z, x)=(0,0)$. It is easy to see that, locally around $z=0, \lim _{\|z\| \rightarrow 0}\left(V_{0}^{\alpha_{0}}(z) / \rho\left(\mathrm{V}_{0}(z)\right) V_{0}^{\alpha_{0}}(z)\right)=$ $1 / \rho(0)<\infty, \lim _{\|z\| \rightarrow 0}\left(V_{*}(z) / V_{0}(z)\right)=\rho(0)<\infty$, and then $\lim _{\|z\| \rightarrow 0}\left(V_{*}^{\alpha_{0}}(z) / \rho\left(V_{0}(z)\right) V_{0}^{\alpha_{0}}(z)\right)=1$, which implies that when $\|z\|$ is small enough, there is a constant $c_{1}$ such that $V_{*}(z)^{\alpha_{0}} \leq\left(c_{0}(1-\epsilon) / c_{1}\right) \rho\left(V_{0}(z)\right) V_{0}^{\alpha_{0}}(z)$. Thus, locally around $(z, x)=(0,0)$, by $(38)$, one has

$$
\dot{\bar{V}}_{c}=-L_{n} Q_{n}^{2-v}-\frac{c_{1}}{2} V_{*}^{\alpha_{0}}+\widetilde{\sigma} \varphi_{n}
$$

Note that

$$
\begin{aligned}
\varphi_{n}(x, \widehat{\sigma}) & =\sum_{j=1}^{n}\left|w_{j}\right|^{(2-v) / r_{j} \beta_{j-1} p_{j-1}} b_{j}\left(\bar{x}_{j}, \widehat{\sigma}\right) \\
& \leq Q_{n}^{2-v} \varphi_{0}(x, \widehat{\sigma}),
\end{aligned}
$$

where $\varphi_{0}=\sum_{j=1}^{n} b_{j}$ is continuous with $\varphi_{0}(0, \widehat{\sigma})=0$ because $b_{j}(0, \widehat{\sigma})=0$. Substituting (40) into (39), it is easy to obtain

$$
\begin{aligned}
\dot{\bar{V}}_{c}= & -\frac{L_{n}}{2} Q_{n}^{2-v}-\frac{c_{1}}{2} V_{*}^{\alpha_{0}} \\
& -L_{n} Q_{n}^{2-v}\left(\frac{1}{2}-\frac{1}{L_{n}}(\sigma+\bar{c}) \varphi_{0}\right),
\end{aligned}
$$

where $\bar{c}$ is a positive constant. By (15) and Lemma 9, then $V_{n}=\sum_{j=1}^{n} \int_{v_{j-1}}^{x_{j}}\left([s]^{\beta_{j-1} p_{j-1}}-\left[v_{j-1}\right]^{\beta_{j-1} p_{j-1}}\right) \mathrm{d} s \leq$ $\sum_{j=1}^{n} 2\left|w_{j}\right|^{2 / r_{j} \beta_{j-1} p_{j-1}}$, which together with (24) and Lemma 5 mean that $V_{n}^{(2-v) / 2} \leq 2 Q_{n}^{2-v}$. Hence, (41) becomes

$$
\dot{\bar{V}}_{c} \leq-\frac{L_{n}}{4} V_{n}^{(2-\nu) / 2}-\frac{c_{1}}{2} V_{*}^{\alpha_{0}}-L_{n} Q_{n}^{2-\nu}\left(\frac{1}{2}-\widetilde{V}\right)
$$

where $\widetilde{V}(x, \widehat{\sigma})=\left(1 / L_{n}\right)(\sigma+\bar{c}) \varphi_{0}(x, \widehat{\sigma})$ is continuous with $\widetilde{V}(0, \widehat{\sigma})=0$. Because $\widetilde{V}$ is continuous and $\widetilde{V}(0, \widehat{\sigma})=0$, it is obvious that there is a constant $0<\varrho \leq 1$ such that $\widetilde{V} \leq 1 / 2$ if $\bar{V}_{c} \leq \varrho$. By Lemma 5 and $\bar{V}_{c} \leq \varrho \leq 1$, choosing $c_{*}=\min \left\{L_{n} / 4, c_{1} / 2\right\}, \alpha=\max \left\{(2-v) / 2, \alpha_{0}\right\}$, we have $\left(L_{n} / 4\right) V_{n}^{(2-\nu) / 2}+\left(c_{1} / 2\right) V_{*}^{\alpha_{0}} \geq c_{*}\left(V_{n}^{\alpha}+V_{*}^{\alpha}\right) \geq c_{*}\left(V_{n}+V_{*}\right)^{\alpha}$. Thus, in a neighborhood $\Omega=\left\{(z, x, \widehat{\sigma}): \bar{V}_{c} \leq \varrho\right\}$, (42) becomes

$$
\dot{\bar{V}}_{c} \leq-c_{*} \bar{V}_{c}^{\alpha}
$$

which implies the local finite-time convergence in $\Omega$ by Lemma 3.

We consider the global finite-time convergence. The finite-time convergence in $\Omega$ has been achieved. We now study the situation outside $\Omega$. When the initial condition is outside $\Omega$, one has $\bar{V}_{c} \geq \varrho$. It is easy to see that there is a constant $c_{2}$ such that $\left(L_{n} / 2\right) V_{n}^{(2-v) / 2}+\left(c_{1} / 2\right) V_{*}^{\alpha_{0}} \geq c_{2} \varrho^{\alpha}$. Let $T_{1}$ be the first time that $(z(t), x(t), \widehat{\sigma}(t))$ intersects the boundary of $\Omega$. Using (37) and the above-mentioned argument, for any $\tau \in\left[0, T_{1}\right)$, one has

$$
\begin{aligned}
V_{c} & (z(0), x(0), \widehat{\sigma}(0)) \\
& \geq V_{c}(z(0), x(0), \widehat{\sigma}(0))-V_{c}(z(\tau), x(\tau), \widehat{\sigma}(\tau)) \\
& =\int_{0}^{\tau}-\dot{V}_{c}(z(s), x(s), \widehat{\sigma}(s)) \mathrm{d} s \\
& =\int_{0}^{\tau}\left(-\dot{\bar{V}}_{c}+\widetilde{\sigma} \varphi_{n}\right) \mathrm{d} s \\
& \geq \int_{0}^{\tau}\left(\frac{L_{n}}{2} V_{n}^{(2-v) / 2}+\frac{c_{1}}{2} V_{*}^{\alpha_{0}}\right) \mathrm{d} s \geq c_{2} \varrho^{\alpha} \tau .
\end{aligned}
$$

If $(z(t), x(t), \widehat{\sigma}(t))$ do not enter $\Omega$ in finite time, then $T_{1}=$ $\infty$. When $\tau \rightarrow T_{1}, c_{2} \varrho^{\alpha} \tau \rightarrow \infty$, while $V_{c}(z(0), x(0), \widehat{\sigma}(0))$ is a finite constant, then (44) leads to a contradiction. So $(z(t), x(t), \widehat{\sigma}(t))$ will reach $\Omega$ in finite time.

Remark 8. We estimate the settling time. In practice, although we do not know the real value of $\sigma(\operatorname{or} \theta)$, we always have its range. Without loss of generality, we assume that $0<\sigma<\Sigma$, where $\Sigma>0$ is a constant. We consider two cases.

Case $1((z(0), x(0), \widehat{\sigma}(0)) \in \Omega)$. By (43), we have

$$
T \leq \frac{\bar{V}_{c}(z(0), x(0), \widehat{\sigma}(0))^{1-\max \left\{(2-\nu) / 2, \alpha_{0}\right\}}}{c_{*}\left(1-\max \left\{(2-\nu) / 2, \alpha_{0}\right\}\right)} .
$$

Case $2((z(0), x(0), \widehat{\sigma}(0)) \notin \Omega)$. Before the state reaches $\Omega$, from (34) and (37), it follows that $\widetilde{\sigma}^{2} \leq 2 V_{c}(z(0), x(0), \widehat{\sigma}(0)) \leq$ $2 \bar{V}_{c}(z(0), x(0), \widehat{\sigma}(0))+(|\widehat{\sigma}(0)|+\Sigma)^{2}$; then $V_{c}(z(0), x(0), \widehat{\sigma}(0)) \leq$ $\bar{V}_{c}(z(0), x(0), \widehat{\sigma}(0))+(1 / 2) \widetilde{\sigma}(0)^{2} \leq 2 \bar{V}_{c}(z(0), x(0), \widehat{\sigma}(0))+$ $(1 / 2)(|\widehat{\sigma}(0)|+\Sigma)^{2}$. Therefore, by $(44),(x, \widehat{\sigma})$ will reach $\Omega$ within $T_{1}$ :

$$
T_{1} \leq \frac{2 \bar{V}_{c}(z(0), x(0), \widehat{\sigma}(0))+(|\widehat{\sigma}(0)|+\Sigma)^{2}}{c_{2} \varrho^{\max \left\{(2-\gamma) / 2, \alpha_{0}\right\}}} .
$$

After $T_{1}$, it will stay in $\Omega$, and it will take

$$
T_{2} \leq \frac{\varrho^{1-\max \left\{(2-\nu) / 2, \alpha_{0}\right\}}}{c_{*}\left(1-\max \left\{(2-\nu) / 2, \alpha_{0}\right\}\right)}
$$

to reach the origin. Therefore, in this case, the settling time can be estimated as $T_{1}+T_{2}$.

Remark 9. Compared with [14], due to the appearance of dynamic and parametric uncertainties, and the weaker condition on nonlinear functions, the main difficulty in this paper is how to skillfully combine Lyapunov function, sign function, backstepping, and FTISS approaches to give the design and rigorous analysis of finite-time controller. 

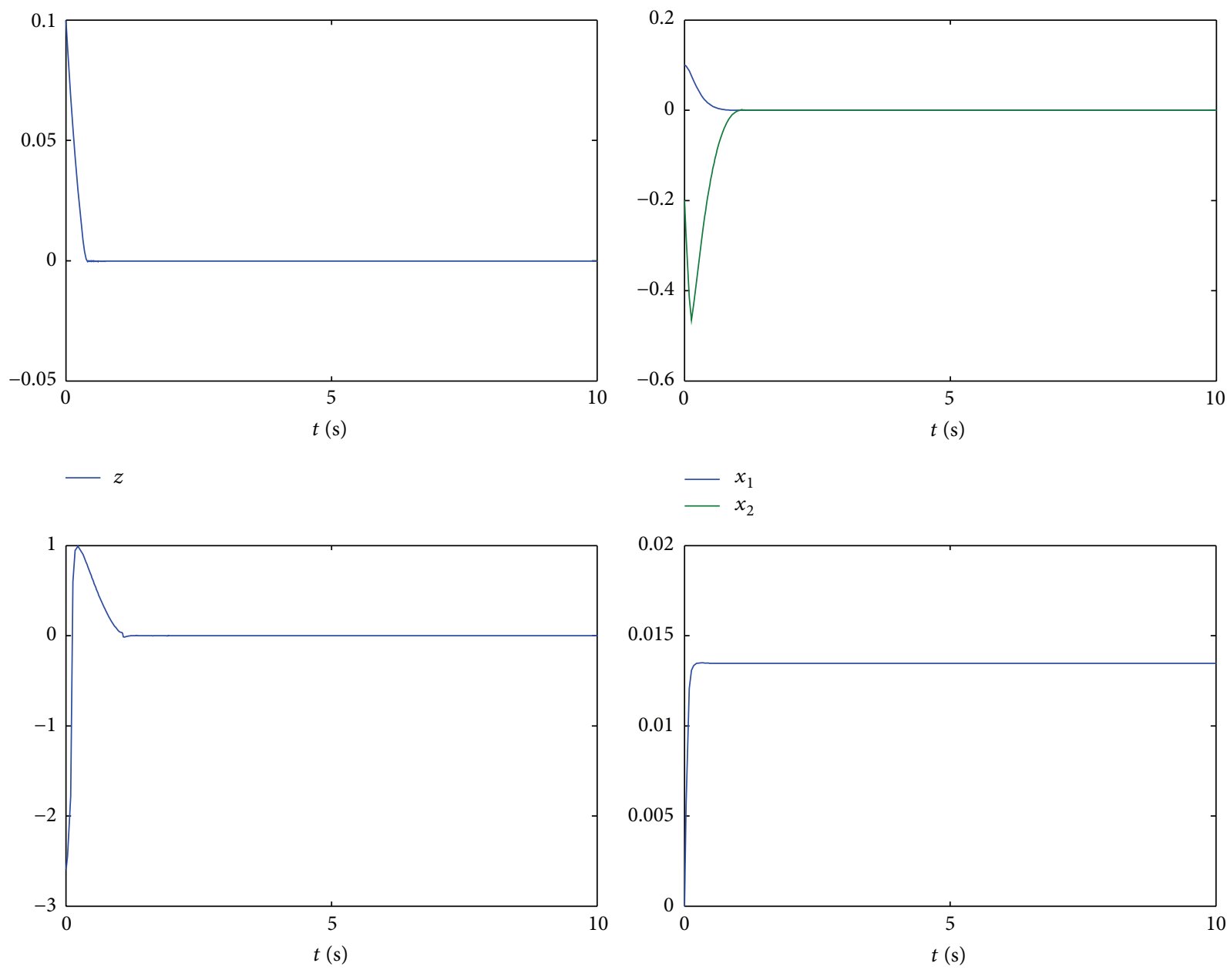

FIGURE 1: Trajectories of $z, x_{1}, x_{2}, u, \widehat{\sigma}$.

\section{A Simulation Example}

Consider a simple system

$$
\begin{aligned}
\dot{z} & =-z^{1 / 3}+x_{1}, \\
\dot{x}_{1} & =x_{2}^{9 / 7}+\theta \sin x_{1} \cos z, \\
\dot{x}_{2} & =u-z^{2} \cos x_{2},
\end{aligned}
$$

where $\theta$ is an unknown parameter and $z$ is the unmeasurable dynamic uncertainty.

Choose $v=2 / 11$; then $r_{1}=1, r_{2}=7 / 11$. By (48), we have $\gamma_{1}=\gamma_{2}=0,\left|\phi_{1}\right|=\left|\theta \sin x_{1} \cos z\right| \leq \theta\left|\sin x_{1}\right| \leq \theta\left|x_{1}\right|^{9 / 11}$, $\left|\phi_{2}\right|=\left|z^{2} \cos x_{2}\right| \leq\|z\|^{2}, \kappa_{1}(\|z\|)=\|z\|^{2}, \kappa_{12}\left(x_{1}\right)=1$, $\kappa_{22}\left(x_{1}, x_{2}\right)=0$, and $\mu_{11}=\mu_{21}=\mu_{22}=0$. Choose $V_{0}(z)=z^{2}$; then $\dot{V}_{0} \leq-(1 / 2) z^{4 / 3}+(1 / 2) x_{1}^{4} \leq-(1 / 2) V_{0}^{2 / 3}+(1 / 2) x_{1}^{4} ; z-$ subsystem is obviously finite-time input-to-state stable with $\gamma_{0}\left(x_{1}\right)=(1 / 2) x_{1}^{4}$. Hence, Assumptions 1-3 hold.
Take $\beta_{0}=1$; then $r_{2} p_{1}+r_{1} \beta_{0} p_{0}=2-v=20 / 11$. Following the design procedure in Section 3, one can obtain the adaptive finite-time controller

$$
\begin{aligned}
u= & -0.2\left(x_{2}^{15 / 7}+2 x_{1}^{15 / 11} \Phi_{1}^{5 / 3}\right)^{1 / 3} \\
& \cdot\left(4+2 \Phi_{1}+3.2 \Phi_{1}^{2}+2 \widehat{\sigma} x_{1}^{4 / 9}\right), \quad \Phi_{1}=4 \widehat{\sigma}^{2} x_{1}^{4}+2 \\
\dot{\hat{\sigma}}= & x_{1}^{20 / 11}+2 x_{1}^{2 / 9}\left(x_{2}^{15 / 7}+2 x_{1}^{15 / 11} \Phi_{1}^{5 / 3}\right)^{4 / 3}
\end{aligned}
$$

Choosing the initial conditions $z(0)=0.1, x_{1}(0)=0.1$, $x_{2}(0)=-0.2$, and $\widehat{\sigma}(0)=0$, we estimate the settling time. Since $\Omega=\left\{(z, x, \widehat{\sigma}): \bar{V}_{c} \leq 0.2\right\}, \rho(s)=8\left(s^{14 / 9}+s^{3}+s^{81 / 78}\right)$, one has $\bar{V}_{c}(z(0), x(0), \widehat{\sigma}(0))=0.0338$, which is in $\Omega$. According to Case 1 in Remark 8 , by $\max \left\{(2-v) / 2, \alpha_{0}\right\}=10 / 11, c_{*}=$ $\min \{0.25,0.1813\}=0.1813$, one has $T \leq 44.5923$.

With $\theta=1$, Figure 1 shows the trajectories of $z, x_{1}, x_{2}, u$, $\widehat{\sigma}$, and one can see that $z, x_{1}, x_{2}$ reach the origin within $T$. 


\section{Conclusions}

By characterizing the dynamic uncertainty with FTISS, under the weaker assumption on nonlinear functions, the problem of adaptive finite-time stabilization for more general highorder nonlinear systems with dynamic and parametric uncertainties is solved.

Some interesting problems are still remaining: (1) For system (2) with possibly nonvanishing disturbances and more general dynamic uncertainty, can a finite-time convergent controller be given? (2) How can we construct output feedback to stabilize system (2) in finite time? (3) In recent years, some results on stochastic nonlinear systems with SISS/SiISS dynamic uncertainty have been obtained, for example, [25-36] and the references therein, but these papers only consider the global asymptotic stabilization. An important problem is whether finite-time stabilization can be obtained for stochastic nonlinear systems with dynamic and parametric uncertainties.

\section{Appendix}

Proof of Proposition 6. We first prove that $V_{i}$ is $\mathscr{C}^{1}$. From (15) and $\Phi_{i-1}\left(\bar{x}_{i-1}, \widehat{\sigma}\right)$ being positive and $\mathscr{C}^{1}$ and $\left[v_{i-1}\right]^{\beta_{i-1} p_{i-1}}$ being $\mathscr{C}^{1}$, then $W_{i}$ is $\mathscr{C}^{1}$, so is $V_{i}$.

Secondly, we prove that $V_{i}$ is positive-definite with respect to $x_{1}, \ldots, x_{i}$. When $x_{i}>v_{i-1}$, by (15), (28), and Lemmas 7 and 9 , one has

$$
\begin{aligned}
W_{i} & \geq 2^{1-\beta_{i-1} p_{i-1}} \int_{v_{i-1}}^{x_{i}}\left(s-v_{i-1}\right)^{\beta_{i-1} p_{i-1}} \mathrm{~d} s \\
& \geq \frac{2^{1-\beta_{i-1} p_{i-1}}}{\beta_{i-1} p_{i-1}+1}\left|x_{i}-v_{i-1}\right|^{\beta_{i-1} p_{i-1}+1} \geq 0 .
\end{aligned}
$$

When $x_{i} \leq v_{i-1}$, it can be shown that (A.1) also holds in a similar way.

From (A.1), the definition of $V_{i}$, and the positive definiteness of $V_{i-1}$ with respect to $x_{1}, \ldots, x_{i-1}$, it is easy to see that $V_{i} \geq 0$ and, for fixed $\widehat{\sigma}, V_{i}\left(\bar{x}_{i}, \widehat{\sigma}\right)=0$ if and only if $\bar{x}_{i}=0$, which implies that $V_{i}$ is positive-definite with respect to $x_{1}, \ldots, x_{i}$.

Finally, we prove (27). From the definition of $V_{i}$ and (23), it follows that

$$
\begin{aligned}
\dot{V}_{i}\left(\bar{x}_{i}, \widehat{\sigma}\right) & \\
\leq & -\gamma_{0}\left(\left|x_{1}\right|\right)\left(\mu\left(x_{1}\right)+1\right)-L_{i-1} Q_{i-1}^{2-\nu} \\
& +\sum_{j=1}^{i-1} \kappa_{1}^{(2-\nu) /\left(2-\nu-r_{j} \beta_{j-1} p_{j-1}\right)}+\sum_{j=2}^{i-1} \kappa_{1}^{(2-\nu) /\left(2-\nu-r_{j}\right)} \\
& +\left(\left|w_{i-1}\right|\left|x_{i}^{p_{i-1}}-v_{i-1}^{p_{i-1}}\right|+\gamma_{i-1}\left|w_{i-1}\right|\left|w_{i}\right|^{1 / \beta_{i-1}}\right) \\
& +\sum_{j=1}^{i} \frac{\partial W_{i}}{\partial x_{j}}\left(x_{i+1}^{p_{i}}+\phi_{i}\right) \\
& +\left(\left(\widetilde{\sigma}+\eta_{i-1}\right) \varphi_{i-1}-\eta_{i-1} \dot{\hat{\sigma}}+\frac{\partial W_{i}}{\partial \widehat{\sigma}}\right)
\end{aligned}
$$

We estimate the last three terms on the right-hand side of (A.2).

(i) By (15), (16), (17), (24), and Lemmas 4 and 9, one has

$$
\begin{aligned}
& \left|w_{i-1}\right|\left|x_{i}^{p_{i-1}}-v_{i-1}^{p_{i-1}}\right|+\gamma_{i-1}\left|w_{i-1}\right|\left|w_{i}\right|^{1 / \beta_{i-1}} \\
& \leq\left(2+\gamma_{i-1}\right)\left|w_{i-1}\right|\left|w_{i}\right|^{1 / \beta_{i-1}} \\
& \leq \frac{L_{i-1}}{4 n} Q_{i-1}^{2-\nu}+l_{i}\left|w_{i}\right|^{(2-v) / r_{i} \beta_{i-1} p_{i-1}}
\end{aligned}
$$

where $l_{i}>0$ is a constant dependent on $\nu, L_{i-1}, \gamma_{i-1}$, $p_{1}, \ldots, p_{i-1}$.

(ii) For $j \leq i-1$, from (15), (17), (24), and Lemmas 4, 8, and 10 , the following holds:

$$
\begin{aligned}
& \left|\frac{\partial\left[v_{i-1}\right]^{\beta_{i-1} p_{i-1}}}{\partial x_{j}}\right| \leq\left|w_{i-1}\right|^{r_{i} p_{i-1} \beta_{i-1} / r_{i-1} \beta_{i-2} p_{i-2}} g\left(\bar{x}_{i-1}, \widehat{\sigma}\right) \\
& \quad+\sum_{k=j+1}^{i-2}\left(\prod_{l=k+1}^{i-1}\left|w_{l}\right|^{\left(r_{l+1} \beta_{l} p_{l}-r_{l} \beta_{l-1} p_{l-1}\right) / r_{l} \beta_{l-1} p_{l-1}}\right) \\
& \cdot\left|w_{k}\right|^{r_{k+1} \beta_{k} p_{k} / r_{k} \beta_{k-1} p_{k-1}} \cdot g\left(\bar{x}_{i-1}, \widehat{\sigma}\right) \\
& +\prod_{l=j+1}^{i-1}\left|w_{l}\right|^{\left(r_{l+1} \beta_{l} p_{l}-r_{l} \beta_{l-1} p_{l-1}\right) / r_{l} \beta_{l-1} p_{l-1}} \mid \partial\left[v_{j}\right]^{\beta_{j} p_{j}} \\
& \quad \cdot g\left(\bar{x}_{j}\right.
\end{aligned} \mid
$$

where $g\left(\bar{x}_{i-1}, \widehat{\sigma}\right)$ is a general $\mathscr{C}^{1}$ nonnegative function that may be implicitly changed in various places and $h_{j, i-1}\left(\bar{x}_{i-1}, \widehat{\sigma}\right)$ is a $\mathscr{C}^{1}$ nonnegative function. From (15), (17), (28), (A.4), and Lemma 9, it follows that

$$
\begin{aligned}
\frac{\partial W_{i}}{\partial x_{j}} & \leq\left|x_{i}-v_{i-1}\right|\left|\frac{\partial\left[v_{i-1}\right]^{\beta_{i-1} p_{i-1}}}{\partial x_{j}}\right| \\
& \leq 2\left|w_{i}\right|^{1 / \beta_{i-1} p_{i-1}} Q_{i-1}^{r_{i} \beta_{i-1} p_{i-1}-r_{j}} h_{j, i-1}, \quad j \leq i-1 .
\end{aligned}
$$


For $i=1, \ldots, n$, by Assumption 2, one has

$$
\begin{aligned}
& \left|\phi_{i}\left(x_{1}, \ldots, x_{i}, z, d\right)\right| \\
& \quad \leq \kappa_{1}(\|z\|)+\gamma_{i}\left|x_{i+1}\right|^{p_{i}} \\
& \quad+\theta \bar{\kappa}_{i 2}\left(x_{1}, \ldots, x_{i}\right) \sum_{l=1}^{i}\left|x_{l}\right|^{\left(r_{i}-v\right) / r_{l}},
\end{aligned}
$$

where $\bar{\kappa}_{i 2}=\sum_{l=1}^{i}\left|x_{l}\right|^{\mu_{i j}} \kappa_{i 2}$ is a $\mathscr{C}$ nonnegative function vanishing at the origin. For $j<i$, by (15), (17), (24), (A.6), and Lemmas 6 and 9,

$$
\begin{aligned}
& x_{j+1}^{p_{j}}+\phi_{j} \leq\left|x_{j+1}^{p_{j}}-v_{j}^{p_{j}}\right|+\left|v_{j}^{p_{j}}\right|+\left|\phi_{j}\right| \leq 2\left|w_{j+1}\right|^{1 / \beta_{j}} \\
& +\left|w_{j}\right|^{\left(r_{j}-v\right) / r_{j} \beta_{j-1} p_{j-1}} \Phi_{j}+\left|\phi_{j}\right| \leq\left(2+\Phi_{j}\right) Q_{j+1}^{r_{j}-v} \\
& +\kappa_{1}+\gamma_{j}\left|x_{j+1}\right|^{p_{j}}+\theta \bar{\kappa}_{j 2}\left(x_{1}, \ldots, x_{j}\right) \sum_{l=1}^{j}\left|x_{l}\right|^{\left(r_{j}-v\right) / r_{l}} \\
& \quad \leq\left(2+\Phi_{j}\right) Q_{j+1}^{r_{j}-v}+\kappa_{1}+\gamma_{j}\left(\left|w_{j+1}\right|^{1 / \beta_{j}}\right. \\
& \left.\quad+\left|w_{j}\right|^{\left(r_{j}-v\right) / r_{j} \beta_{j-1} p_{j-1}} \Phi_{j}\right)+\theta \bar{\kappa}_{j 2}\left(x_{1}, \ldots, x_{j}\right) \\
& \quad \cdot \sum_{l=1}^{j}\left(\left|w_{l}\right|^{\left(r_{j}-v\right) / r_{l} \beta_{l-1} p_{l-1}}\right. \\
& \left.\quad+\left|w_{l-1}\right|^{\left(r_{j}-v\right) / r_{l-1} \beta_{l-2} p_{l-2}} \Phi_{l-1}^{\left(r_{j}-v\right) / r_{l} p_{l-1}}\right) .
\end{aligned}
$$

From (16), (A.5), (A.7), Lemma 4, and $(2-v) / r_{i} \leq(2-v) / r_{n}$,

$$
\begin{aligned}
& \sum_{j=1}^{i-1} \frac{\partial W_{i}}{\partial x_{j}}\left(x_{j+1}^{p_{j}}+\phi_{j}\right) \leq 2\left|w_{i}\right|^{1 / \beta_{i-1} p_{i-1}}\left(Q_{i}^{r_{i} \beta_{i-1} p_{i-1}-\nu} \sum_{j=1}^{i-1} h_{j, i-1}(2\right. \\
& \left.\quad+\Phi_{j}\right)+\sum_{j=1}^{i-1} Q_{i}^{r_{i} \beta_{i-1} p_{i-1}-r_{j}} h_{j, i-1}\left(\kappa_{1}+\gamma_{j}\left(\left|w_{j+1}\right|^{1 / \beta_{j}}\right.\right. \\
& \left.\quad+\left|w_{j}\right|^{\left(r_{j}-v\right) / r_{j} \beta_{j-1} p_{j-1}} \Phi_{j}\right)+\theta \bar{\kappa}_{j 2}\left(x_{1}, \ldots, x_{j}\right) \\
& \left.\left.\quad \cdot \sum_{l=1}^{j}\left(\left|w_{l}\right|^{\left(r_{j}-v\right) / r_{l} \beta_{l-1} p_{l-1}}+\left|w_{l-1}\right|^{\left(r_{j}-v\right) / r_{l-1} \beta_{l-2} p_{l-2}} \Phi_{l-1}^{\left(r_{j}-v\right) / r_{l} p_{l-1}}\right)\right)\right) \\
& \leq \frac{L_{i-1}}{4 n} Q_{i}^{2-\gamma}+\left|w_{i}\right|^{(2-v) / r_{i} \beta_{i-1} p_{i-1}}\left(\psi_{i, 1}+\sigma b_{i, 1}\right)+\kappa_{1}^{(2-\nu) /\left(2-v-r_{i}\right)},
\end{aligned}
$$

where $\psi_{i, 1}\left(\bar{x}_{i-1}, \widehat{\sigma}\right)$ and $b_{i, 1}\left(\bar{x}_{i-1}, \widehat{\sigma}\right)$ are $\mathscr{C}$ nonnegative functions with $b_{i, 1}(0, \widehat{\sigma})=0$. It follows from (15), (16), (17), (24), (28), (A.6), and Lemmas 4 and 6 that

$$
\begin{aligned}
& \frac{\partial W_{i}}{\partial x_{i}}\left(x_{i+1}^{p_{i}}+\phi_{i}\right) \leq\left|w_{i}\right|\left|x_{i+1}^{p_{i}}-v_{i}^{p_{i}}\right|+w_{i} v_{i}^{p_{i}}+\left|w_{i}\right|\left(\kappa_{1}\right. \\
& +\gamma_{i}\left(\left|w_{i+1}\right|^{1 / \beta_{i}}+\left|w_{i}\right|^{\left(r_{i}-v\right) / r_{i} \beta_{i-1} p_{i-1}} \Phi_{i}\right)+\theta \bar{\kappa}_{i 2}\left(x_{1}\right. \\
& \left.\quad \ldots, x_{i}\right) \sum_{l=1}^{i}\left(\left|w_{l}\right|^{\left(r_{i}-v\right) / r_{l} \beta_{l-1} p_{l-1}}\right. \\
& \left.\left.+\left|w_{l-1}\right|^{\left(r_{j}-v\right) / r_{l-1} \beta_{l-2} p_{l-2}} \Phi_{l-1}^{\left(r_{j}-v\right) / r_{l} p_{l-1}}\right)\right) \leq \frac{L_{i-1}}{4 n} Q_{i}^{2-\nu} \\
& +\left|w_{i}\right|\left|x_{i+1}^{p_{i}}-v_{i}^{p_{i}}\right|+w_{i} v_{i}^{p_{i}}+\left|w_{i}\right|^{(2-v) / r_{i} \beta_{i-1} p_{i-1}}\left(\psi_{i, 2}\right. \\
& \left.+\sigma b_{i, 2}\right)+\kappa_{1}^{(2-v) /\left(2-\nu-r_{i} \beta_{i-1} p_{i-1}\right)}+\gamma_{i}\left|w_{i}\right|\left|w_{i+1}\right|^{1 / \beta_{i}} \\
& +\gamma_{i}\left|w_{i}\right|^{(2-v) / r_{i} \beta_{i-1} p_{i-1}} \Phi_{i},
\end{aligned}
$$

where $\psi_{i, 2}\left(\bar{x}_{i}, \widehat{\sigma}\right)$ and $b_{i, 2}\left(\bar{x}_{i}, \widehat{\sigma}\right)$ are $\mathscr{C}$ nonnegative functions with $b_{i, 2}(0, \widehat{\sigma})=0$. Combining (A.8) with (A.9) leads to

$$
\begin{aligned}
\sum_{j=1}^{i} \frac{\partial W_{i}}{\partial x_{j}}\left(x_{j+1}^{p_{j}}+\phi_{j}\right) \leq & \left|w_{i}\right|\left|x_{i+1}^{p_{i}}-v_{i}^{p_{i}}\right|+w_{i} v_{i}^{p_{i}} \\
& +\frac{L_{i-1}}{2 n} Q_{i}^{2-\nu} \\
& +\left|w_{i}\right|^{(2-\nu) / r_{i} \beta_{i-1} p_{i-1}}\left(\psi_{i}+\sigma b_{i}\right) \\
& +\kappa_{1}^{(2-\nu) /\left(2-\nu-r_{i}\right)} \\
& +\kappa_{1}^{(2-\nu) /\left(2-\nu-r_{i} \beta_{i-1} p_{i-1}\right)} \\
& +\gamma_{i}\left|w_{i}\right|\left|w_{i+1}\right|^{1 / \beta_{i}} \\
& +\gamma_{i}\left|w_{i}\right|^{(2-\nu) / r_{i} \beta_{i-1} p_{i-1}} \Phi_{i}
\end{aligned}
$$

where $\psi_{i}=\psi_{i, 1}+\psi_{i, 2}, b_{i}=b_{i, 1}+b_{i, 2}$.

(iii) For the last term of (A.2), from (17), (28), and Lemma 8 , there is a $\mathscr{C}^{1}$ nonnegative function $\delta_{j}\left(\bar{x}_{j}, \widehat{\sigma}\right)$ such that $\left|\partial\left[v_{j}\right]^{\beta_{j} p_{j}} / \partial \widehat{\sigma}\right| \leq \delta_{j}\left(\bar{x}_{j}, \widehat{\sigma}\right)(j \leq i-1)$. By (24), (25), and Lemma 4,

$$
\begin{aligned}
\left(\widetilde{\sigma}+\eta_{i-1}\right) \varphi_{i-1}-\eta_{i-1} \dot{\hat{\sigma}}+\frac{\partial W_{i}}{\partial \widehat{\sigma}} \dot{\hat{\sigma}} \\
=\left(\widetilde{\sigma}+\eta_{i}+\frac{\partial W_{i}}{\partial \widehat{\sigma}}\right)\left(\varphi_{i}-\left|w_{i}\right|^{(2-\nu) / r_{i} \beta_{i-1} p_{i-1}} b_{i}\right) \\
\quad-\eta_{i} \dot{\hat{\sigma}}
\end{aligned}
$$




$$
\begin{aligned}
= & \left(\widetilde{\sigma}+\eta_{i}\right) \varphi_{i}-\left(\widetilde{\sigma}+\eta_{i}\right)\left|w_{i}\right|^{(2-v) / r_{i} \beta_{i-1} p_{i-1}} b_{i} \\
& +\frac{\partial W_{i}}{\partial \widehat{\sigma}} \varphi_{i}-\frac{\partial W_{i}}{\partial \widehat{\sigma}}\left|w_{i}\right|^{(2-v) / r_{i} \beta_{i-1} p_{i-1}} b_{i}-\eta_{i} \dot{\widehat{\sigma}} \\
\leq & \left(\widetilde{\sigma}+\eta_{i}\right) \varphi_{i} \\
& -\left|w_{i}\right|^{(2-v) / r_{i} \beta_{i-1} p_{i-1}} b_{i}\left(\widetilde{\sigma}+\eta_{i}+\delta_{i-1}\left|w_{i}\right|^{1 / \beta_{i-1} p_{i-1}}\right) \\
& -\eta_{i} \dot{\hat{\sigma}}+\left|w_{i}\right|^{1 / \beta_{i-1} p_{i-1}} \delta_{i-1} \sum_{j=1}^{i}\left|w_{j}\right|^{(2-v) / r_{j} \beta_{j-1} p_{j-1}} b_{j} \\
\leq & \frac{L_{i-1}}{4 n} Q_{i}^{2-v}+\left|w_{i}\right|^{(2-v) / r_{i} \beta_{i-1} p_{i-1}}\left(\phi_{i}^{0}-\widetilde{\sigma} b_{i}\right) \\
& +\left(\widetilde{\sigma}+\eta_{i}\right) \varphi_{i}-\eta_{i} \dot{\widehat{\sigma}},
\end{aligned}
$$

where $\phi_{i}^{0}\left(\bar{x}_{i}, \widehat{\sigma}\right)$ is $\mathscr{C}$ nonnegative function;

$$
\begin{aligned}
\varphi_{i}\left(\bar{x}_{i}, \widehat{\sigma}\right)= & \varphi_{i-1}\left(\bar{x}_{i-1}, \widehat{\sigma}\right) \\
& +\left|w_{i}\right|^{(2-v) / r_{i} \beta_{i-1} p_{i-1}} b_{i}\left(\bar{x}_{i}, \widehat{\sigma}\right) .
\end{aligned}
$$

Substituting (A.3), (A.10), and (A.11) into (A.2), one has

$$
\begin{aligned}
\dot{V}_{i}\left(\bar{x}_{i}, \widehat{\sigma}\right) \leq & -\gamma_{0}\left(\left|x_{1}\right|\right)\left(\mu\left(x_{1}\right)+1\right) \\
& -\frac{n-1}{n} L_{i-1} Q_{i-1}^{2-\nu}+\left|w_{i}\right|\left|x_{i+1}^{p_{i}}-v_{i}^{p_{i}}\right| \\
& +\left(\widetilde{\sigma}+\eta_{i}\right) \varphi_{i}-\eta_{i} \dot{\hat{\sigma}} \\
& +\sum_{j=1}^{i} \kappa_{1}^{(2-v) /\left(2-v-r_{j} \beta_{j-1} p_{j-1}\right)} \\
& +\sum_{j=2}^{i} \kappa_{1}^{(2-\nu) /\left(2-v-r_{j}\right)}+\gamma_{i}\left|w_{i}\right|\left|w_{i+1}\right|^{1 / \beta_{i}} \\
& +w_{i} v_{i}^{p_{i}}+\gamma_{i}\left|w_{i}\right|^{(2-v) / r_{i} \beta_{i-1} p_{i-1}} \Phi_{i} \\
& +\left|w_{i}\right|^{(2-\nu) / r_{i} \beta_{i-1} p_{i-1}}\left(l_{i}+\psi_{i}+\phi_{i}^{0}+\hat{\sigma} b_{i}\right) .
\end{aligned}
$$

Using Lemma 8 , one can choose a $\mathscr{C}^{1}$ positive function

$$
\begin{aligned}
& \Phi_{i} \\
& \geq \frac{1}{1-\gamma_{i}}\left(\frac{n-1}{n} L_{i-1}+l_{i}+\psi_{i}+\phi_{i}^{0}+\left(1+\widehat{\sigma}^{2}\right) b_{i}\right),
\end{aligned}
$$

such that

$$
\begin{aligned}
w_{i} v_{i}^{p_{i}} & +\left|w_{i}\right|^{(2-v) / r_{i} \beta_{i-1} p_{i-1}}\left(l_{i}+\psi_{i}+\phi_{i}^{0}+\widehat{\sigma} b_{i}\right) \\
& +\gamma_{i}\left|w_{i}\right|^{(2-\nu) / r_{i} \beta_{i-1} p_{i-1}} \Phi_{i} \\
\leq & -L_{i}\left|w_{i}\right|^{(2-\nu) / r_{i} \beta_{i-1} p_{i-1}},
\end{aligned}
$$

where $L_{i}=((n-1) / n) L_{i-1}$. Substituting (A.15) into (A.13) leads to (27).

\section{Competing Interests}

The authors declare that they have no competing interests.

\section{Acknowledgments}

This work was supported by the National Natural Science Foundation of China (nos. 61273125 and 61673242).

\section{References}

[1] V. T. Haimo, "Finite time controllers," SIAM Journal on Control and Optimization, vol. 24, no. 4, pp. 760-770, 1986.

[2] S. P. Bhat and D. S. Bernstein, "Finite-time stability of continuous autonomous systems," SIAM Journal on Control and Optimization, vol. 38, no. 3, pp. 751-766, 2000.

[3] E. Moulay and W. Perruquetti, "Finite time stability and stabilization of a class of continuous systems," Journal of Mathematical Analysis and Applications, vol. 323, no. 2, pp. 1430-1443, 2006.

[4] W. M. Haddad, S. G. Nersesov, and L. Du, "Finite-time stability for time-varying nonlinear dynamical systems," in Proceedings of the American Control Conference (ACC '08), pp. 4135-4139, Seattle, Wash, USA, June 2008.

[5] P. Krishnamurthy, F. Khorrami, and R. S. Chandra, "Global high-gain-based observer and backstepping controller for generalized output-feedback canonical form," IEEE Transactions on Automatic Control, vol. 48, no. 12, pp. 2277-2284, 2003.

[6] L. Praly and Z. P. Jiang, "Linear output feedback with dynamic high gain for nonlinear systems," Systems and Control Letters, vol. 53, no. 2, pp. 107-116, 2004.

[7] X..-Q. Huang, W. Lin, and B. Yang, "Global finite-time stabilization of a class of uncertain nonlinear systems," Automatica, vol. 41, no. 5, pp. 881-888, 2005.

[8] X.-F. Zhang, L. Baron, Q.-R. Liu, and E.-K. Boukas, “Design of stabilizing controllers with a dynamic gain for feedforward nonlinear time-delay systems," IEEE Transactions on Automatic Control, vol. 56, no. 3, pp. 692-697, 2011.

[9] X.-F. Zhang, G. Feng, and Y.-G. Sun, "Finite-time stabilization by state feedback control for a class of time-varying nonlinear systems," Automatica, vol. 48, no. 3, pp. 499-504, 2012.

[10] J. Li, C.-J. Qian, and S.-H. Ding, "Global finite-time stabilisation by output feedback for a class of uncertain nonlinear systems," International Journal of Control, vol. 83, no. 11, pp. 2241-2252, 2010.

[11] J. Wang, J. Xie, and F. Gao, "Global finite-time stabilization for a class of uncertain high-order nonlinear systems," Abstract and Applied Analysis, vol. 2014, Article ID 384713, 7 pages, 2014.

[12] K.-M. Zhang and X.-H. Zhang, "Finite-time stabilisation for high-order nonlinear systems with low-order and high-order nonlinearities," International Journal of Control, vol. 88, no. 8, pp. 1576-1585, 2015.

[13] Y.-G. Hong, J.-K. Wang, and D.-Z. Cheng, "Adaptive finite-time control of nonlinear systems with parametric uncertainty," IEEE Transactions on Automatic Control, vol. 51, no. 5, pp. 858-862, 2006.

[14] Z.-Y. Sun, L.-R. Xue, and K. Zhang, "A new approach to finitetime adaptive stabilization of high-order uncertain nonlinear system," Automatica, vol. 58, no. 8, pp. 60-66, 2015. 
[15] J. Tsinias, "Partial-state global stabilization for general triangular systems," Systems \& Control Letters, vol. 24, no. 2, pp. 139-145, 1995.

[16] Z.-P. Jiang and L. Praly, "Design of robust adaptive controllers for nonlinear systems with dynamic uncertainties," Automatica, vol. 34, no. 7, pp. 825-840, 1998.

[17] Z.-P. Jiang and I. Mareels, "Robust nonlinear integral control," IEEE Transactions on Automatic Control, vol. 46, no. 8, pp. 13361342, 2001.

[18] Z.-P. Jiang, I. Mareels, D. J. Hill, and J. Huang, "A unifying framework for global regulation via nonlinear output feedback: from ISS to iISS," IEEE Transactions on Automatic Control, vol. 49, no. 4, pp. 549-562, 2004.

[19] J. Huang, Nonlinear Output Regulation: Theory and Applications, vol. 8 of Advances in Design and Control, Society for Industrial and Applied Mathematics, Philadelphia, Pa, USA, 2004.

[20] Y.-G. Hong and Z.-P. Jiang, "Finite-time stabilization of nonlinear systems with parametric and dynamic uncertainties," IEEE Transactions on Automatic Control, vol. 51, no. 12, pp. 1950-1956, 2006.

[21] Y.-G. Hong, Z.-P. Jiang, and G. Feng, "Finite-time input-to-state stability and applications to finite-time control design," SIAM Journal on Control and Optimization, vol. 48, no. 7, pp. 43954418, 2010.

[22] C.-J. Qian and W. Lin, "A continuous feedback approach to global strong stabilization of nonlinear systems," IEEE Transactions on Automatic Control, vol. 46, no. 7, pp. 1061-1079, 2001.

[23] W. Lin and C.-J. Qian, "Adaptive control of nonlinearly parameterized systems: the smooth feedback case," IEEE Transactions on Automatic Control, vol. 47, no. 8, pp. 1249-1266, 2002.

[24] Z.-Y. Sun, X.-H. Zhang, and X.-J. Xie, "Continuous global stabilisation of high-order time-delay nonlinear systems," International Journal of Control, vol. 86, no. 6, pp. 994-1007, 2013.

[25] C. Tang and T. Başar, "Stochastic stability of singularly perturbed nonlinear systems," in Proceedings of the 40th IEEE Conference on Decision and Control (CDC '01), pp. 399-404, December 2001.

[26] H. Ito and Y. Nishimura, "Stability of stochastic nonlinear systems in cascade with not necessarily unbounded decay rates," Automatica, vol. 62, no. 9, pp. 51-64, 2015.

[27] S.-J. Liu, J.-F. Zhang, and Z.-P. Jiang, "Decentralized adaptive output-feedback stabilization for large-scale stochastic nonlinear systems," Automatica, vol. 43, no. 2, pp. 238-251, 2007.

[28] S.-J. Liu and J.-F. Zhang, "Output-feedback control of a class of stochastic nonlinear systems with linearly bounded unmeasurable states," International Journal of Robust and Nonlinear Control, vol. 18, no. 6, pp. 665-687, 2008.

[29] X. Yu, X.-J. Xie, and N. Duan, "Small-gain control method for stochastic nonlinear systems with stochastic iISS inverse dynamics," Automatica, vol. 46, no. 11, pp. 1790-1798, 2010.

[30] X. Yu and X.-J. Xie, "Output feedback regulation of stochastic nonlinear systems with stochastic iISS inverse dynamics," IEEE Transactions on Automatic Control, vol. 55, no. 2, pp. 304-320, 2010.

[31] X.-J. Xie, N. Duan, and X. Yu, "State-feedback control of high-order stochastic nonlinear systems with SiISS inverse dynamics," IEEE Transactions on Automatic Control, vol. 56, no. 8, pp. 1921-1926, 2011.

[32] N. Duan and X.-J. Xie, "Further results on output-feedback stabilization for a class of stochastic nonlinear systems," IEEE
Transactions on Automatic Control, vol. 56, no. 5, pp. 1208-1213, 2011.

[33] N. Duan, X. Yu, and X.-J. Xie, “Output feedback control using small-gain conditions for stochastic nonlinear systems with SiISS inverse dynamics," International Journal of Control, vol. 84, no. 1, pp. 47-56, 2011.

[34] L. Liu and X.-J. Xie, "State-feedback stabilization for stochastic high-order nonlinear systems with SISS inverse dynamics," Asian Journal of Control, vol. 14, no. 1, pp. 207-216, 2012.

[35] C.-R. Zhao and X.-J. Xie, “Output feedback stabilization using small-gain method and reduced-order observer for stochastic nonlinear systems," IEEE Transactions on Automatic Control, vol. 58, no. 2, pp. 523-529, 2013.

[36] L. Liu, S. Yin, H.-J. Gao, F. Alsaadi, and T. Hayat, "Adaptive partial-state feedback control for stochastic high-order nonlinear systems with stochastic input-to-state stable inverse dynamics," Automatica, vol. 51, no. 1, pp. 285-291, 2015. 


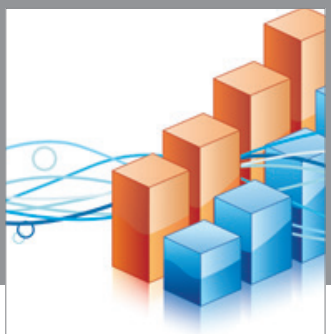

Advances in

Operations Research

vatem alat4

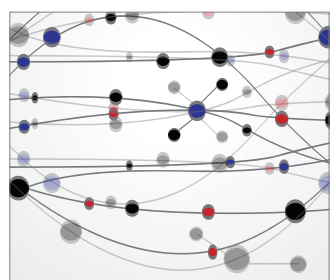

\section{The Scientific} World Journal
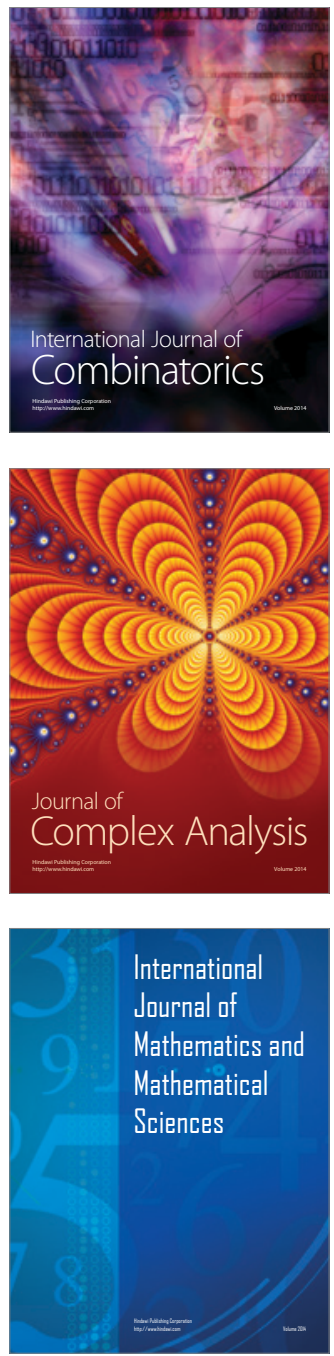
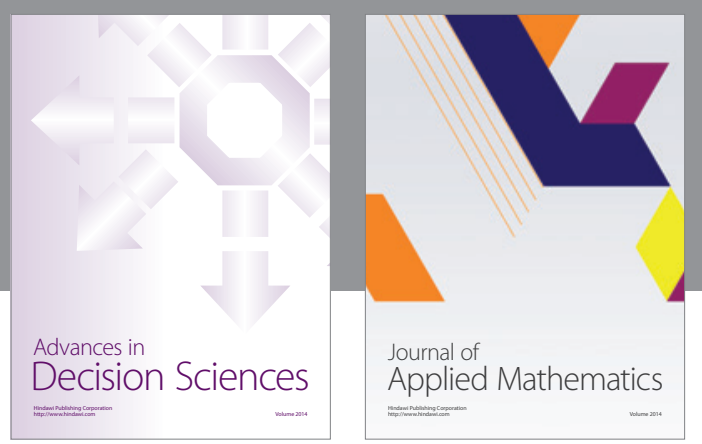

Algebra

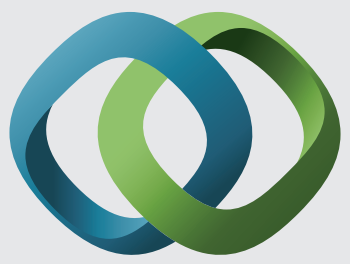

\section{Hindawi}

Submit your manuscripts at

http://www.hindawi.com
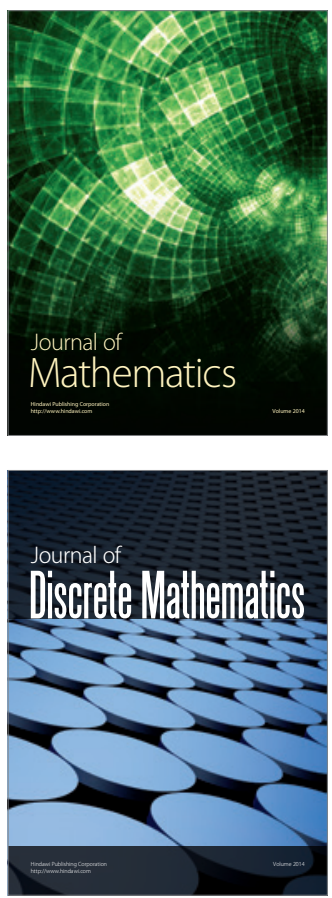

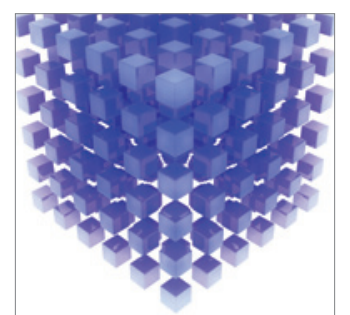

Mathematical Problems in Engineering
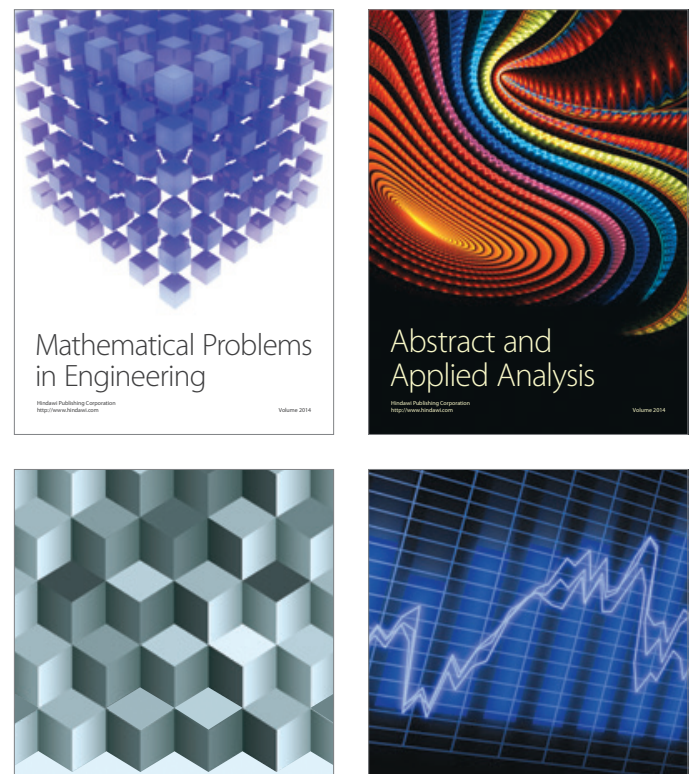

Journal of

Function Spaces

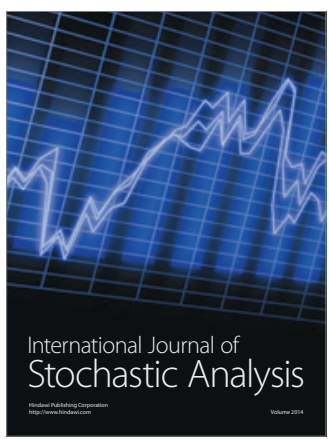

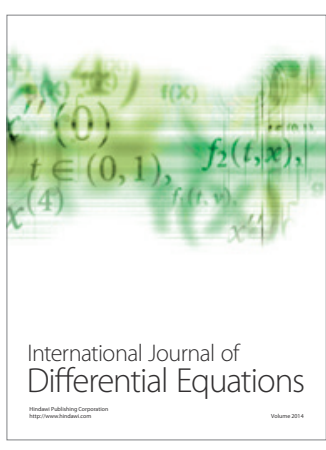
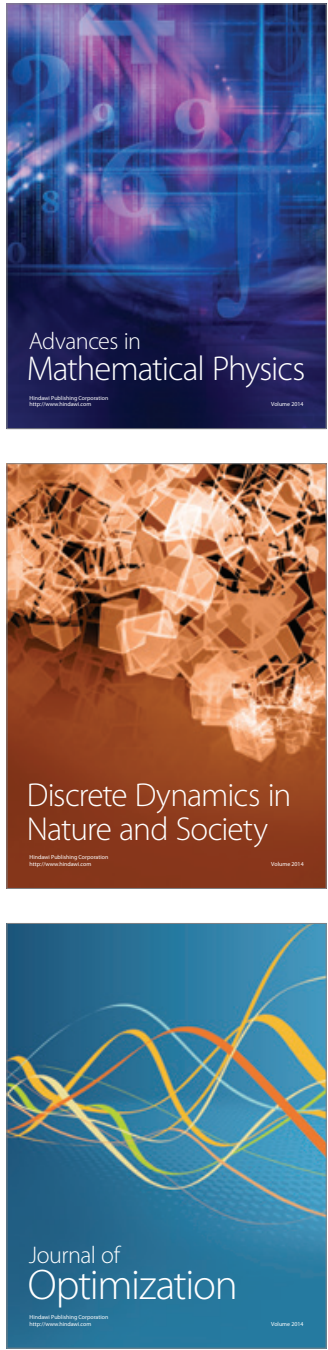